\title{
Expression Analysis of Circulating miR-22, miR-122, miR- 217 and miR-367 as Promising Biomarkers of Acute Lymphoblastic Leukemia
}

Fatemeh hosseinpour-soleimani

Shiraz University of Medical Sciences

Gholamreza Khamisipour ( $\sim$ khamisipourgholamreza@gmail.com )

Bushehr University of Medical Sciences

Zahra Derakhshan

Bushehr University of Medical Sciences

Bahram Ahmadi

Bushehr University of Medical Sciences

\section{Research Article}

Keywords: MicroRNA, Biomarker, Acute Lymphoblastic Leukemia, serum, miR-217, miR-367, miR-22, miR-122

Posted Date: December 1st, 2021

DOI: https://doi.org/10.21203/rs.3.rs-1097151/v1

License: (c) (i) This work is licensed under a Creative Commons Attribution 4.0 International License. Read Full License 


\section{Abstract \\ Background}

Currently, the role of serum-based biomarkers such as microRNAs in cancer diagnosis has been extensively established. This study aimed to determine expression levels of bioinformatically selected miRNAs and whether they can be used as biomarkers or a new therapeutic target in patients with Acute Lymphoblastic Leukemia (ALL).

\section{Materials and Methods}

The expression levels of serum miR-22, miR-122, miR-217, and miR-367 in 21 ALL patients and 21 healthy controls were measured using quantitative real-time PCR. The receiver operating characteristic (ROC) curve and the associated area under the curve (AUC) was used to assess candidate miRNAs' diagnostic value as a biomarker.

\section{Results}

The results showed that miR-217 was markedly decreased in patients with ALL compared to controls. Moreover, miR-22, miR-122, and miR-367 were found to be upregulated. Furthermore, ROC analysis showed that serum miR217 and miR-367 could differentiate ALL patients from the healthy individuals, while miR-22 has approximate discriminatory power that requires further investigation.

\section{Conclusion}

Collectively, the results suggested that miR-217 may play a tumor suppressor role in ALL, whereas miR-22, miR-122, and miR-367 could function as an oncogene. Overall, miR-22, miR-217, and miR-367 could be considered possible biomarkers for the early diagnosis of ALL.

\section{Introduction}

Acute lymphoblastic leukemia (ALL) is a neoplastic abnormality of the lymphoid lineage known primarily as a childhood disease, with $75 \%$ occurrence in children under the age of 10 years (1). The underlying mechanisms of leukemia are numerous genetic mutations that lead to chromosomal abnormalities and malignant cell division and proliferation composed of immature and dysfunctional lymphocytes (2). Although inappropriate gene function and altered gene expression patterns are a hallmark of ALL, but not enough to cause leukemia and it is most likely caused by interaction between exogenous and endogenous factors, genetic or some hereditary factors, and chance (3).

Careful assessment of prognosis and risk factors and rapid diagnosis is essential for ALL management and successful treatment (1). Due to the lack of sensitivity and specificity of early-stage cancer diagnosis and being aggressive, tumor biopsy-based diagnostic methods are less commonly used to monitor the treatment of patients (4). More recently, the role of bio-fluids-based biomarkers and liquid biopsy in cancer diagnosis has been extensively established due to their non-invasive protocols and ease to acquire (5). Since the discovery of the first miRNA (a small non-coding single-stranded RNA molecule) called lin-4 (Lineage-4) in 1993 (6), numerous studies have shown that inappropriate miRNA expression is associated with many human diseases, including cancer $(7,8)$. Interestingly, 
they can be as "oncomiRs (oncogenic miRNAs)" or "tumor suppressor miRNAs" and affect the significant carcinogenic pathways that are often involved in apoptosis, cell proliferation, angiogenesis, metastasis, and drug resistance (7). In addition, miRNAs could be considered as a biomarker of disease due to their tiny structure and stability under various conditions such as boiling, multiple freeze-thaw cycles, and pH changes (8), as well as secretion into extracellular biofluids including serum and plasma (9), saliva (10) urine, seminal fluid, breast milk, cerebrospinal fluid, bronchial lavage and also tears (11).

It has recently been shown that miRNA expression profiles vary across different types of leukemia and are associated with survival and prognosis (7). In this study, according to the genes and signaling pathways involved in the ALL, four related miRNAs (MiR-22, miR-122, miR-217, and miR-367) were selected using reliable predictive databases and - previous studies. Despite the therapeutic and diagnostic advances, there is still a frequent failure in ALL. Hence, there is an indispensable need to look at new diagnostic and treatment goals. Thus, this study aimed to assess the potential impact of miRNAs as serum biomarkers and or new therapeutic targets in patients with ALL.

\section{Material And Methods \\ 2.1. Subjects}

The study protocol was approved by the Medical Ethics Committee of Bushehr University of Medical Sciences, Bushehr, Iran (no. IR.BPUMS.REC.1397.059, approved October 22, 2019), and all tests were performed according to the relevant guidelines with prior informed consent from all participants. The current cross-sectional study included 21 patients with newly diagnosed ALL before any administration of chemotherapy, and 21 healthy controls without underlying disease of matched gender and age. Moreover, samples of the same ALL patients were enrolled in the study after one month of routine treatment. The serum of all specimens was separated immediately after blood collection and divided into aliquots to $1.5-\mathrm{mL}$ cryotubes, then stored at $-80^{\circ} \mathrm{C}$ until further use.

\subsection{Bioinformatics analysis}

In the present study, the possible role of miRNAs in ALL was investigated. For this purpose, several genes and signaling pathways involved in developing this disease were identified based on the literature. After that, the interaction of these pathways and genes with the expression of miRNAs was searched in databases. For further investigation, the DIANA TOOLS - miR Path v. three databases (http://www.microrna.gr/miRPathv3) and KEGG (Kyoto Encyclopedia of Genes and Genomes) pathway analysis were employed to predicts the candidate miRNAs targets of a particular function in diseases or pathways, based on three algorithms: Tarbase, MicroT-CDS, and Targetscan. Besides, miRWalk (http://mirwalk.uni-hd.de/) was used to get more information on interactions between miRNAs and target genes. Subsequently, the desired microRNAs were selected focusing on KEGG pathways and based on the results of the predictive sites.

\section{3. miRNA isolation and cDNA synthesis}

In the pre-cDNA synthesis steps, $200 \mu \mathrm{L}$ serum was utilized to directly withdraw RNA from the serum using Tween20 buffer as described by Asaga S and Hoon DS (12). Samples were transferred from $-80^{\circ} \mathrm{C}$ to $-20^{\circ} \mathrm{C}$ to prevent shock and kept at room temperature subsequently. They were centrifuged at 15,000 rpm for $10 \mathrm{~min}$, then $10 \mu \mathrm{L}$ of each sample's supernatant was incubated with $10 \mu \mathrm{L}$ of Tween-20 buffer for 10 minutes at $70^{\circ} \mathrm{C}$ in a Dry Bath (Dolphin Co., India). After that, the samples were immediately placed on ice for Tween-20 settling and then centrifuged at 11,000 rpm for 2 minutes. Then, cDNA was synthesized using a reverse transcription kit according to the Oligo-dT method (BonYakhteh Co., Iran) as recommended in the manufacturer's instructions. The purity and 
quantity of cDNA was assessed spectrophotometrically as 260/280 nm and 260/230 nm ratios, using NanoDrop DS-11 (DeNovix Inc., Wilmington, DE USA).

\subsection{Quantitative Real-time PCR (qRT-PCR)}

The qRT-PCR was performed in duplicate using SYBR Green Master Mix (Yektatajhiz CO., Iran) and specific primers for miR-22, 122, 217, and 367 (BonYakhteh Co., Iran) by the ABI StepOnePlus RT-PCR system (Applied Biosystems, USA). $0.5 \mu \mathrm{L}$ of each forward and reverse primer were used for each reaction. The primer sequences are listed in Table.1.

The temperature and schedule were set as one primary denaturation at $95^{\circ} \mathrm{C}$ for 2 min followed by 45 cycles of amplification at $95^{\circ} \mathrm{C}$ for $5 \mathrm{~s}$, and finally, $60^{\circ} \mathrm{C}$ for $30 \mathrm{~s}$ to confirm amplification specificity. Moreover, non-template control (NTC) was used for each primer to ensure the target gene amplification and lack of contamination during operation.

\subsection{Statistical analysis}

Data quality control was performed using boxplot to identify and modify outliers for the miRNAs expression levels in controls and patients. The relative quantification of target miRNAs expression was calculated using the $2^{-\Delta \Delta C t}$ method (13), and cycle thresholds (Ct) values were normalized to miR-17 as an internal control. The Shapiro-Wilk test evaluated the normality of the data distribution. The unpaired T-test or Mann-Whitney test were performed to compare the differences in miRNAs expression between the healthy controls and patients and also paired T-test or

Wilcoxon for comparison between the patient's group before and after treatment, using statistical package for social sciences (SPSS) 16 (SPSS Inc., Chicago, IL, USA) and GraphPad Prism 7 (GraphPad Prism Software, Inc. San Diego CA, USA). Furthermore, the receiver operating characteristic (ROC) curve and the associated area under the curve (AUC) was used to determine diagnostic value of candidate miRNAs' as a biomarker. A p-value less than 0.05 was considered statistically significant for each test.

\section{Results}

\subsection{Characteristic of patients}

The study population included 21 patients with newly diagnosed ALL, consisting of 13 men (61.9\%) and 8 women (38.1\%), and 21 age- and gender-matched healthy controls without underlying disease. Table.2 summarizes the demographic and laboratory characteristics of the patients at the time of diagnosis. According to the results, expression of miR-22 was significantly higher in the adult group, while there was no significant difference in the relative expression levels of other candidate miRNAs between men and women, children and adults, and the ALL subtypes. Moreover, no statistically significant correlation was observed between the expression of miRNAs with age, platelets count, white and red blood cell count, and hemoglobin.

\subsection{Differential expression of serum miRNAs in newly diagnosed ALL patients}

Levels of serum miRNAs were assessed by qPCR to evaluate their role in the diagnosis of ALL. As shown in Fig. 1AC, relative serum miR-22 and miR-367 expression increased significantly and up-regulated in the newly diagnosed ALL group compared to control $(P=0.01$ and 0.002 , respectively). Although the serum miR-122 levels showed increased expression, it was not statistically significant $(P=0.06)$. 
Moreover, we found that the relative levels of serum miR-217 expression were markedly decreased, and it was downregulated in the newly diagnosed ALL group compared to the controls ( $P<0.0001$, Fig. 1D).

\subsection{The changes in serum miRNAs expression in ALL patients following treatment}

One month after the first routine treatment of the patients, their blood samples were examined to evaluate the changes in the expression level of the miRNAs with the mentioned methods. The results demonstrated that the expression levels of miR-22, miR-122, and miR-367 in the follow-up groups were decreased compared to the first examination ( $P=0.05, P=0.78$, and $P=0.005$, respectively, Fig. $2 A-C)$. Interestingly, the expression level of miR-217 was significantly higher in the follow-up group than in the preliminary assessment (P=0.01, Fig. 2D).

\subsection{The diagnostic performance of candidate miRNAs for ALL patients}

ROC curve analysis was assessed to determine the specificity and sensitivity of each miRNA as a possible biomarker in the diagnosis and follow-up of ALL patients. To distinguish the newly diagnosed ALL group from the control, an AUC for miR-22, miR-122, miR-217 and miR-367 was found to be 0.7 with a sensitivity of $61 \%$ and a specificity of $71 \%(P=0.03,95 \%$ Cl: $0.69-0.94), 0.62$ with a sensitivity of $33 \%$ and a specificity of $100 \%(P=0.17,95 \%$ Cl: $0.45-0.79), 0.81$ with a sensitivity of $95 \%$ and a specificity of $61 \%(P=0,95 \% \mathrm{Cl}: 0.69-0.94)$ and 0.8 with a sensitivity of $80 \%$ and a specificity of $81 \%$ ( $P=0.001,95 \% \mathrm{Cl}$ : $0.65-0.94)$; respectively (Fig. 3A-D).

Moreover, AUC in follow-up group for miR-22, miR-122, miR-217 and miR-367 was found to be 0.57 with a sensitivity of $38 \%$ and a specificity of $85 \%(P=0.43,95 \% \mathrm{Cl}: 0.39-0.74), 0.6$ with a sensitivity of $38 \%$ and a specificity of $95 \%$ $(P=0.25,95 \% \mathrm{Cl}: 0.65-0.94), 0.65$ with a sensitivity of $80 \%$ and a specificity of $61 \%(P=0.08,95 \% \mathrm{Cl}: 0.48-0.83)$ and 0.55 with a sensitivity of $42 \%$ and a specificity of $76 \%(P=0.53,95 \%$ Cl: $0.37-0.73)$; respectively (Fig. 4A-D).

\subsection{Logistic Regression Results}

The data of qPCR were used for binary logistic regression. According to the results of ROC curves, miR-217 and miR367 were selected to simultaneously evaluate the role of biomarkers due to their appropriate sensitivity and specificity and higher Yuden index in newly diagnosed ALL patients. The mean logit $(P)$ value of the ALL group was found to be 0.83 and significantly higher than that of the control group, $0.17(P<0.0001)$. The predicted values of logistic regression analysis showed that the combined ROC analysis of these two miRNA revealed an increased AUC value of 0.95 , with a sensitivity of $95 \%$, a specificity of $90 \%$, and a Yuden index of 0.85 ( $P=0,95 \%$ Cl: $0.88-1.01$; Fig. 5). A logit (P) of 0.50 was used for optimal cutoff by which ALL and controls were distinguished (Table.3).

\section{Discussion}

MiRNAs are currently well defined as small regulatory molecules whose significant role is in post-transcriptional regulation of their target genes and play different crucial roles in physiological and pathological cellular processes (14). To date, the expression profile and dysregulation of microRNAs has been studied in more than a dozen disease, particularly various types of human cancer (15-17). Since the aberrant expression of serum miRNAs in cancer was for the first time described (18), their role in tumorigenesis has been highly regarded by researchers. Importantly, miRNAs are effective as biomarkers in classifying and tracing the source tissue of unknown primary and poorly differentiated cancers $(19,20)$. These findings emphasize the diagnostic value of miRNAs in cancers and also suggest their therapeutic role. 
To the best of our knowledge, this study is the first report on the assessment of serum miR-22, miR-122, miR-217, and miR-367 in ALL and the use of combine miR-217 and miR-367 as biomarkers in this disease. Specifically, we found that the levels of miR-22, miR-122, and 367 were upregulated in ALL patients compared with controls, and additionally, miR-217 was markedly reduced. The analysis of ROC data revealed that serum miR-217 and miR-367 alone could be effective as a biomarker for early detection of ALL, while miR-22 has approximate discriminatory power that requires further investigation. Moreover, the combination of miR-217 and miR-367 further increased the diagnostic value and could distinguish newly diagnosed ALL patients from healthy controls.

Previous studies have examined the different roles of these miRNAs in various cancers. They have been described as double-faced molecules that can act as both a tumor suppressor and an oncogene. Bar $\mathrm{N}$ et al. demonstrated that overexpression of miR-22 can affect the tumor suppressor, i.e., PTENand its downstream pathways PI3/AKT, thus inhibit AKT proliferative and anti-apoptotic activity (21). Another study by Song et al. reported that miR-22 is a crucial regulator for hematopoiesis maintenance, and it was found to be upregulated in myelodysplastic syndrome and leukemia associated with a poor prognosis. In this study, the TET-2 gene was mentioned as a target of miR-22, which its deletion is involved in hematologic malignancy (22). Given that PTENactivity is lost in many types of cancer, leading to increased cell survival and cell cycle progression (23), as well as the relationship between TET-2 and hematopoietic cell proliferation (24), it seems that miR-22 upregulation may lead to ALL due to its effect on these genes. Conversely, miR-22 was mentioned as a tumor suppressor in a study on acute myeloid leukemia (AML), which affects the MYC pathways (25). Due to the various functions of miRNAs in different tissues, a discrepancy in the results is conceivable. Furthermore, we found that the expression level of miR-22 was not significantly related to the patient's sex and laboratory information but was higher in the adult group, which could be related to the severity of ALL in adults. In a similar study on AML, a relationship between miR-22 and sex, platelet count, and disease subtypes was not reported, whereas a significant relationship was found with white blood cells and cytogenetic (26).

Similar to the results of our study, Manfe et al. mentioned miR-122 as an oncogene that enhances the anti-apoptotic pathway of AKT / P53 and represses chemotherapy-induced cytotoxicity in cutaneous T-cell lymphoma (27). Fornari et al. also evaluated the association between miR-122 and apoptosis and identified cyclin G1 as a target of miR-122 (28). By contrast, Yang et al. demonstrated that expression of miR-122 in AML patients was significantly lower than in the healthy group, and it may function as a tumor suppressor. Moreover, they showed that the induction of miR122 could inhibit cell proliferation in both HL60 and K562 cell lines of AML compared to the control cell line (29). Consistent with the data of Zhang et al. on gastric cancer, in the present study, no significant relationship was observed between miR-122 expression levels and laboratory or demographic findings of patients (30).

According to the present study results, a marked downregulation of miR-217 was observed that confirmed the tumor suppressor action of miR-217. Moreover, we found a significant difference in the expression level of miR-217 in the post-treatment group compared to before treatment, which may be due to the effects of the treatment process. Our results are consistent with a previous study that demonstrated that the expression of miR-217 is decreased in colorectal cancer with the effect of the MAPK pathway, which regulates tumor growth and increases apoptosis (31). Other studies have identified AKT3 (32), Runx2 (33), E2F3 (34), and KRAS (35) as targets of miR-217. The interaction of miR-217 and these genes appear to be involved in pathogenicity in ALL. By contrast, in the study by Yebenes et al., miR-217 was mentioned as an oncogene that increases specifically in the germinal center of lymph nodes. According to this study, miR-217 reduces the expression of genes that respond to DNA damage and its repair and stabilizes the BCL-6 expression (36). Although the ROC analysis in a present study showed that miR-217 is favorable as a biomarker for diagnosing and following up the patients with ALL, it could be more potent in diagnosing. In line 
with these results, in a study by Yan et al., the ROC analysis revealed that miR-217 could consider as a promising biomarker with high sensitivity and specificity in AML (37).

In the previous study, miR-367 has been identified as a regulator of embryonic stem cell self-renewality and pluripotency, which induces the trait of being stemness in the cancer cell and contributes to the progression and spread of cancer (38). Consistent with the present study's data, Kaid et al. showed that miR-367 plays an oncogenic role in medulloblastoma cells by affecting OCT4 transcription factor, leading to poor survival and poor prognosis (39). In another similar study, miR-367 was mentioned as an oncogene that targets tumor suppressor KLF4. It could be blocked at both mRNA and protein levels and its subsequent pathways, BAX and caspase-3, leading to suppress apoptosis (40). Most studies indicated that miR-367 acts primarily as an oncogene. However, contrary to the present study's findings, it was shown that the expression of miR-367 was significantly reduced in gastric cancer tissues (41) and oral carcinoma cells (42).

According to the results of ROC analysis and based on the sensitivity, specificity, and Yuden index, we found that among the studied miRNAs, miR-217 and miR-367 have a more desirable ability to diagnose ALL, and a combination of these two miRNAs could reach a higher sensitivity and specificity. The limitations of our study were; limited access to newly diagnosed ALL patients, small sample sizes, and lack of access to patients' follow-up samples after the treatment process, which was due to the patient's death or referral to out-of-reach medical centers.

\section{Conclusion}

In conclusion, our results suggest that miR-217 and miR-367 could consider as potential new biomarkers for ALL diagnosing, as well as a new therapeutic target. Further comprehensive studies are warranted to confirm these results and investigate the possible mechanisms and role of genes associated with these miRNAs in ALL.

\section{Abbreviations}

ALL: Acute lymphoblastic leukemia

RNA: Ribonucleic acid

cDNA: Complementary deoxyribonucleic acid

Rpm: Revolutions per minute

PCR: Polymerase chain reaction

QRT-PCR: Quantitative Real-time polymerase chain reaction

Ct: Cycle of threshold

ROC: Receiver operating characteristic

AUC: Area under the receiver operating characteristic Curve

PTEN: Phosphatase and tensin homolog

PI3: phosphatidylinositol 3 
TET-2: Ten-Eleven Translocation-2

AML: Acute myeloid leukemia

Myc: myelocytomatosis

MAPK: Mitogen-activated protein kinase

RUNX2: Runt-related transcription factor 2

E2F3: E2F transcription factor 3

KRAS: Kirsten rat sarcoma viral oncogene homolog

BCL-6: B-cell lymphoma protein 6

OCT4: Octamer-binding transcription factor 4

KLF4: Krüppel-like factor 4

BAX: B-cell Lymphoma protein-2 associated X protein

\section{Declarations}

\section{Author's Contributions:}

F-HS: Performed material preparation and all experiments, analyzed the data and wrote the initial draft of the manuscript. Z-D and B-A: Contributed to bioinformatics analysis and revised the manuscript. Gh-Kh: Contributed to concept and design, financial support, and revised the manuscript. All authors read and approved the final manuscript.

\section{Acknowledgement:}

The authors would like to gratefully thank Ms. Elham Mohseni Nasab for her assistance in collecting patient's samples. This work was based on the Research Project No. 844, as the Master dissertation of Fatemeh HosseinpourSoleimani, financed by the Research Council of Bushehr University of Medical Sciences, Bushehr, Iran.

\section{Funding details:}

The present research was supported by an MSc grant provided by Bushehr University of Medical Sciences, Bushehr, Iran (project number: 844). The results presented in this publication are part of the Master dissertation of Fatemeh Hosseinpour-Soleimani.

\section{Conflict of interest:}

The authors declare no conflicts of interest relevant to this article.

\section{Ethics approval:}

The study protocol was approved by the Medical Ethics Committee of Bushehr University of Medical Sciences, Bushehr, Iran (no. IR.BPUMS.REC.1397.059, approved October 22, 2019) and all tests were performed according to 
the relevant guidelines and comply with the Declaration of Helsinki. Informed consent was obtained from all individual participants included in the study.

\section{Data availability:}

The datasets generated and/or analyzed during the current study are available from the corresponding author on reasonable request.

\section{References}

1. Terwilliger T, Abdul-Hay M (2017) Acute lymphoblastic leukemia: a comprehensive review and 2017 update. Blood cancer journal 7(6):e577-e

2. Malard F, Mohty M (2020) Acute lymphoblastic leukaemia. The Lancet 395(10230):1146-1162

3. Shahriari M, Jafari M, Khalafi M, Ramezani M, Maki M, Soleimani FH et al (2018) Pre-and post-birth causes of acute lymphoblastic leukemia.International Journal of Cancer Management. ; 11(6)

4. Valihrach L, Androvic P, Kubista M (2020) Circulating miRNA analysis for cancer diagnostics and therapy. Mol Aspects Med 72:100825

5. Ruhen O, Meehan K (2019) Tumor-Derived Extracellular Vesicles as a Novel Source of Protein Biomarkers for Cancer Diagnosis and Monitoring. Proteomics 19(1-2):1800155

6. Lee RC, Feinbaum RL, Ambros V (1993) The C. elegans heterochronic gene lin-4 encodes small RNAs with antisense complementarity to lin-14. Cell 75(5):843-854

7. Allegra A, Alonci A, Campo S, Penna G, Petrungaro A, Gerace D et al (2012) Circulating microRNAs: new biomarkers in diagnosis, prognosis and treatment of cancer. Int J Oncol 41(6):1897-1912

8. Chen X, Ba Y, Ma L, Cai X, Yin Y, Wang K et al (2008) Characterization of microRNAs in serum: a novel class of biomarkers for diagnosis of cancer and other diseases. Cell Res 18(10):997-1006

9. Hunter MP, Ismail N, Zhang X, Aguda BD, Lee EJ, Yu L et al (2008) Detection of microRNA expression in human peripheral blood microvesicles. PLoS ONE 3(11):e3694

10. Gallo A, Tandon M, Alevizos I, Illei GG (2012) The majority of microRNAs detectable in serum and saliva is concentrated in exosomes. PLoS ONE 7(3):e30679

11. Weber JA, Baxter DH, Zhang S, Huang DY, Huang KH, Lee MJ et al (2010) The MicroRNA Spectrum in 12 Body Fluids. Clin Chem 56(11):1733-1741

12. Asaga S, Hoon DS (2013) Direct serum assay for microRNA in cancer patients. Springer, Circulating MicroRNAs, pp 147-155

13. Livak KJ, Schmittgen TD (2001) Analysis of relative gene expression data using real-time quantitative PCR and the $2-\Delta \Delta$ CT method. Methods 25(4):402-408

14. Iorio MV, Croce CM (2009) MicroRNAs in cancer: small molecules with a huge impact. J Clin Oncol 27(34):5848

15. Marco MD, Ramassone A, Pagotto S, Anastasiadou E, Veronese A, Visone R (2018) MicroRNAs in autoimmunity and hematological malignancies. Int J Mol Sci 19(10):3139

16. Friedman JM, Jones PA (2009) MicroRNAs: critical mediators of differentiation, development and disease. Swiss Med Wkly 139(33-34):466

17. Long H, Wang X, Chen Y, Wang L, Zhao M, Lu Q (2018) Dysregulation of microRNAs in autoimmune diseases: Pathogenesis, biomarkers and potential therapeutic targets. Cancer Lett 428:90-103 
18. Lawrie C, Gal S, Dunlop H, Pushkaran B, Liggins A, Pulford K et al (2008) Detection of elevated levels of tumourassociated microRNAs in serum of patients with diffuse large B-cell lymphoma. Br J Haematol 141(5):672

19. Rosenfeld N, Aharonov R, Meiri E, Rosenwald S, Spector Y, Zepeniuk M et al (2008) MicroRNAs accurately identify cancer tissue origin. Nat Biotechnol 26(4):462-469

20. Lu J, Getz G, Miska EA, Alvarez-Saavedra E, Lamb J, Peck D et al (2005) MicroRNA expression profiles classify human cancers. Nature 435(7043):834-838

21. Bar N, Dikstein R (2010) miR-22 forms a regulatory loop in PTEN/AKT pathway and modulates signaling kinetics. PLoS ONE 5(5):e10859

22. Song S, Ito K, Ala U, Kats L, Webster K, Sun S et al (2013) The oncogenic microRNA miR-22 targets the TET2 tumor suppressor to promote hematopoietic stem cell self-renewal and transformation. Cell Stem Cell 13(1):87-101

23. Gutierrez A, Sanda T, Grebliunaite R, Carracedo A, Salmena L, Ahn Y et al (2009) High frequency of PTEN, PI3K, and AKT abnormalities in T-cell acute lymphoblastic leukemia. Blood, The Journal of the American Society of Hematology 114(3):647-650

24. Li Z, Cai X, Cai C-L, Wang J, Zhang W, Petersen BE et al (2011) Deletion of Tet2 in mice leads to dysregulated hematopoietic stem cells and subsequent development of myeloid malignancies. Blood, The Journal of the American Society of Hematology 118(17):4509-4518

25. Jiang X, Hu C, Arnovitz S, Bugno J, Yu M, Zuo Z et al (2016) miR-22 has a potent anti-tumour role with therapeutic potential in acute myeloid leukaemia. Nat Commun 7(1):1-15

26. Qu H, Zheng G, Cheng S, Xie W, Liu X, Tao Y et al (2020) Serum miR-22 is a novel prognostic marker for acute myeloid leukemia.Journal of Clinical Laboratory Analysis. :e23370

27. Manfè V, Biskup E, Rosbjerg A, Kamstrup M, Skov AG, Lerche CM et al (2012) miR-122 Regulates p53/Akt Signalling and the Chemotherapy-Induced Apoptosis in Cutaneous T-Cell Lymphoma. PLoS ONE 7(1):e29541

28. Fornari F, Gramantieri L, Giovannini C, Veronese A, Ferracin M, Sabbioni S et al (2009) MiR-122/cyclin G1 interaction modulates p53 activity and affects doxorubicin sensitivity of human hepatocarcinoma cells. Cancer Res 69(14):5761-5767

29. Yang J, Yuan Y, Yang X, Hong Z, Yang L (2017) Decreased expression of microRNA-122 is associated with an unfavorable prognosis in childhood acute myeloid leukemia and function analysis indicates a therapeutic potential. Pathology-Research and Practice 213(9):1166-1172

30. Zhang Y, Huang H, Zhang Y, Liao N (2019) Combined detection of serum MiR-221-3p and MiR-122-5p expression in diagnosis and prognosis of gastric cancer. J Gastric Cancer 19(3):315-328

31. Zhang N, Lu C, Chen L (2016) miR-217 regulates tumor growth and apoptosis by targeting the MAPK signaling pathway in colorectal cancer. Oncology letters 12(6):4589-4597

32. Lin Y, Cheng K, Wang T, Xie Q, Chen M, Chen Q et al (2017) miR-217 inhibits proliferation, migration, and invasion via targeting AKT3 in thyroid cancer. Biomed Pharmacother 95:1718-1724

33. Zhu Y, Zhao H, Feng L, Xu S (2016) MicroRNA-217 inhibits cell proliferation and invasion by targeting Runx2 in human glioma. American journal of translational research 8(3):1482

34. Su J, Wang Q, Liu Y, Zhong M (2014) miR-217 inhibits invasion of hepatocellular carcinoma cells through direct suppression of E2F3. Mol Cell Biochem 392(1-2):289-296

35. Zhao W-G, Yu S-N, Lu Z-H, Ma Y-H, Gu Y-M, Chen J (2010) The miR-217 microRNA functions as a potential tumor suppressor in pancreatic ductal adenocarcinoma by targeting KRAS. Carcinogenesis 31(10):1726-1733

Page 10/16 
36. de Yébenes VG, Bartolomé-Izquierdo N, Nogales-Cadenas R, Pérez-Durán P, Mur SM, Martínez N et al (2014) miR217 is an oncogene that enhances the germinal center reaction. Blood 124(2):229-239

37. Yan J, Wu G, Chen J, Xiong L, Chen G, Li P (2018) Downregulated miR-217 expression predicts a poor outcome in acute myeloid leukemia. Cancer Biomarkers 22(1):73-78

38. Zhang Z, Xiang D, Heriyanto F, Gao Y, Qian Z, Wu W-S (2013) Dissecting the roles of miR-302/367 cluster in cellular reprogramming using TALE-based repressor and TALEN. Stem cell reports 1(3):218-225

39. Kaid C, Silva PB, Cortez BA, Rodini CO, Semedo-Kuriki P, Okamoto OK (2015) miR-367 promotes proliferation and stem-like traits in medulloblastoma cells. Cancer Sci 106(9):1188-1195

40. Wang G-C, He Q-Y, Tong D-K, Wang C-F, Liu K, Ding C et al (2016) MiR-367 negatively regulates apoptosis induced by adriamycin in osteosarcoma cells by targeting KLF4. Journal of bone oncology 5(2):51-56

41. Bin Z, Dedong H, Xiangjie F, Hongwei X, Qinghui Y (2015) The microRNA-367 inhibits the invasion and metastasis of gastric cancer by directly repressing Rab23. Genetic testing and molecular biomarkers 19(2):6974

42. Sun H, Feng X (2020) MicroRNA-367 directly targets PIK3R3 to inhibit proliferation and invasion of oral carcinoma cells.Bioscience Reports. ; 40(5)

\section{Tables}

Table. 1 List of primer sequences used for RT-PCR analysis in this study.

\begin{tabular}{|lll|}
\hline Primers & Sequence $\left(5^{\prime}{ }^{\prime} \mathbf{3}^{\prime}\right)$ & TM $\left({ }^{\circ} \mathrm{C}\right)$ \\
\hline miR-17 & TGA CAA AGT GCT TAC AGT & 60 \\
miR-22 & TGG AGT TCT TCA GTG & 60 \\
miR-122 & AGG CTG GAG TGT GAC A & 60 \\
miR-217 & AGG CTA CTG CAT CAG G & 60 \\
miR-367 & AAG CAC TTT AGC AAT GG & 60 \\
\hline
\end{tabular}

Table.2 Laboratory information of patients and its relationship with the expression level of miRNAs at the time of diagnosis $(n=21)$ 


\begin{tabular}{|c|c|c|c|c|c|c|c|c|c|}
\hline $\begin{array}{l}\text { Laboratory } \\
\text { parameters }\end{array}$ & No. & $\mathrm{miR}-22 \Delta \mathrm{Ct}$ & $\begin{array}{l}p \\
\text { value }\end{array}$ & $\begin{array}{l}\mathrm{miR}_{\Delta \mathrm{Ct}}-122 \\
\end{array}$ & $\begin{array}{l}p \\
\text { value }\end{array}$ & $\begin{array}{l}\operatorname{miR}-217 \\
\Delta \mathrm{Ct}\end{array}$ & $\begin{array}{l}\mathrm{p} \\
\text { value }\end{array}$ & $\begin{array}{l}\operatorname{miR}-367 \\
\Delta \mathrm{Ct}\end{array}$ & $\begin{array}{l}p \\
\text { value }\end{array}$ \\
\hline \multicolumn{10}{|l|}{ Gender } \\
\hline Male & 13 & $14.26 \pm 2.55$ & \multirow[t]{2}{*}{0.14} & $13.03 \pm 2.15$ & \multirow[t]{2}{*}{0.23} & $16.63 \pm 3.11$ & \multirow[t]{2}{*}{0.28} & $10.97 \pm 2.19$ & \multirow[t]{2}{*}{0.05} \\
\hline Female & 8 & $15.91 \pm 2.26$ & & $14.29 \pm 2.54$ & & $18.11 \pm 2.63$ & & $12.76 \pm 1.70$ & \\
\hline \multicolumn{10}{|l|}{ Age } \\
\hline$<15$ & 16 & $15.55 \pm 2.01$ & \multirow[t]{2}{*}{0.02} & $13.86 \pm 2.04$ & \multirow[t]{2}{*}{0.22} & $17.54 \pm 2.55$ & \multirow[t]{2}{*}{0.35} & $11.92 \pm 1.84$ & \multirow[t]{2}{*}{0.10} \\
\hline$\geq 15$ & 5 & $12.78 \pm 3.10$ & & $12.39 \pm 3.06$ & & $16.06 \pm 4.16$ & & $10.13 \pm 2.57$ & \\
\hline \multicolumn{10}{|l|}{ WBC counts } \\
\hline$<15000$ & 18 & $15.19 \pm 2.57$ & \multirow[t]{2}{*}{0.64} & $13.33 \pm 2.37$ & \multirow[t]{2}{*}{0.41} & $16.89 \pm 2.79$ & \multirow[t]{2}{*}{0.25} & $11.51 \pm 2.29$ & \multirow[t]{2}{*}{0.93} \\
\hline$\geq 15000$ & 3 & $13.05 \pm 1.27$ & & $14.59 \pm 2.07$ & & $19.05 \pm 3.97$ & & $11.39 \pm 0.55$ & \\
\hline \multicolumn{10}{|c|}{ Platelet counts } \\
\hline$<100000$ & 16 & $14.76 \pm 2.71$ & \multirow[t]{2}{*}{0.16} & $13.40 \pm 2.48$ & \multirow[t]{2}{*}{0.60} & $17.51 \pm 2.97$ & \multirow[t]{2}{*}{0.24} & $11.47 \pm 2.17$ & \multirow[t]{2}{*}{0.92} \\
\hline$\geq 100000$ & 5 & $15.65 \pm 0.66$ & & $14.17 \pm 1.13$ & & $15.31 \pm 2.61$ & & $11.61 \pm 2.18$ & \\
\hline \multicolumn{10}{|c|}{ Hemoglobin concentration } \\
\hline$<10$ & 18 & $14.93 \pm 2.70$ & \multirow[t]{2}{*}{0.86} & $13.72 \pm 2.36$ & \multirow[t]{2}{*}{0.48} & $16.97 \pm 2.97$ & 0.40 & $11.47 \pm 2.29$ & \multirow[t]{2}{*}{0.89} \\
\hline$\geq 10$ & 3 & $14.65 \pm 1.24$ & & $12.26 \pm 2.01$ & & $18.55 \pm 3.14$ & & $11.65 \pm 0.26$ & \\
\hline \multicolumn{10}{|c|}{ ALL subtype according to CD markers } \\
\hline Pre-B ALL & 15 & $14.79 \pm 2.89$ & \multirow[t]{3}{*}{0.52} & $13.66 \pm 2.52$ & \multirow[t]{3}{*}{0.90} & $16.59 \pm 3.20$ & \multirow[t]{3}{*}{0.30} & $12.05 \pm 1.45$ & \multirow[t]{3}{*}{0.15} \\
\hline Pro-B ALL & 3 & $16.31 \pm 0.60$ & & $13.16 \pm 0.34$ & & $18.13 \pm 2.06$ & & $9.77 \pm 1.59$ & \\
\hline T-ALL & 3 & $13.94 \pm 0.72$ & & $13.11 \pm 3.0$ & & $19.31 \pm 1.14$ & & $10.42 \pm 4.38$ & \\
\hline
\end{tabular}

Table. 3 Distribution of newly diagnosed ALL group and control, using logit $(p)=0.50$ as a cutoff value

\begin{tabular}{|llll|}
\hline Parameter & Control group & ALL group & Total \\
\hline Test positive* $^{\star}$ & 2 & 20 & 22 \\
Test negative** & 19 & 1 & 20 \\
Total & 21 & 21 & 42 \\
*logit $(P)>0.5,{ }^{\star *} \operatorname{logit}(P)<0.5$ & & \\
\hline
\end{tabular}

\section{Figures}




\section{Figure 1}

Levels of serum miRNAs were assessed by qPCR to evaluate their role in the diagnosis of ALL. As shown in Fig. 1A$C$, relative serum miR-22 and miR-367 expression increased significantly and up-regulated in the newly diagnosed ALL group compared to control ( $P=0.01$ and 0.002 , respectively). Although the serum miR-122 levels showed increased expression, it was not statistically significant $(P=0.06)$. Moreover, we found that the relative levels of serum miR-217 expression were markedly decreased, and it was down-regulated in the newly diagnosed ALL group compared to the controls $(P<0.0001$, Fig. 1D).

\section{Figure 2}

The results demonstrated that the expression levels of miR-22, miR-122, and miR-367 in the follow-up groups were decreased compared to the first examination $(P=0.05, P=0.78$, and $P=0.005$, respectively, Fig. 2A-C). Interestingly, the expression level of miR-217 was significantly higher in the follow-up group than in the preliminary assessment $(P=0.01$, Fig. 2D). 


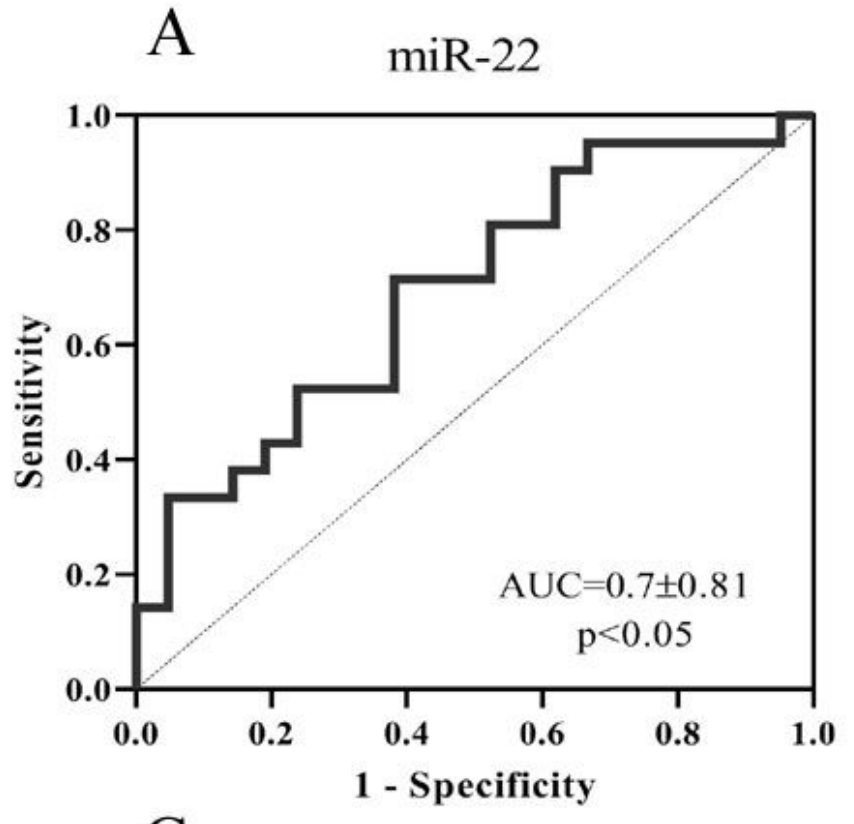

$\operatorname{miR}-367$

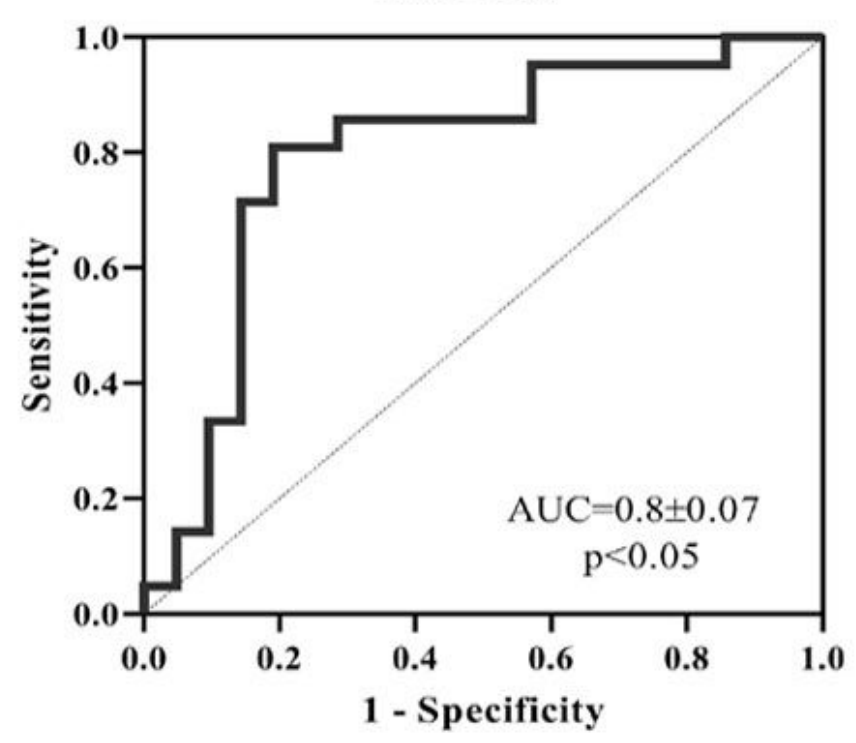

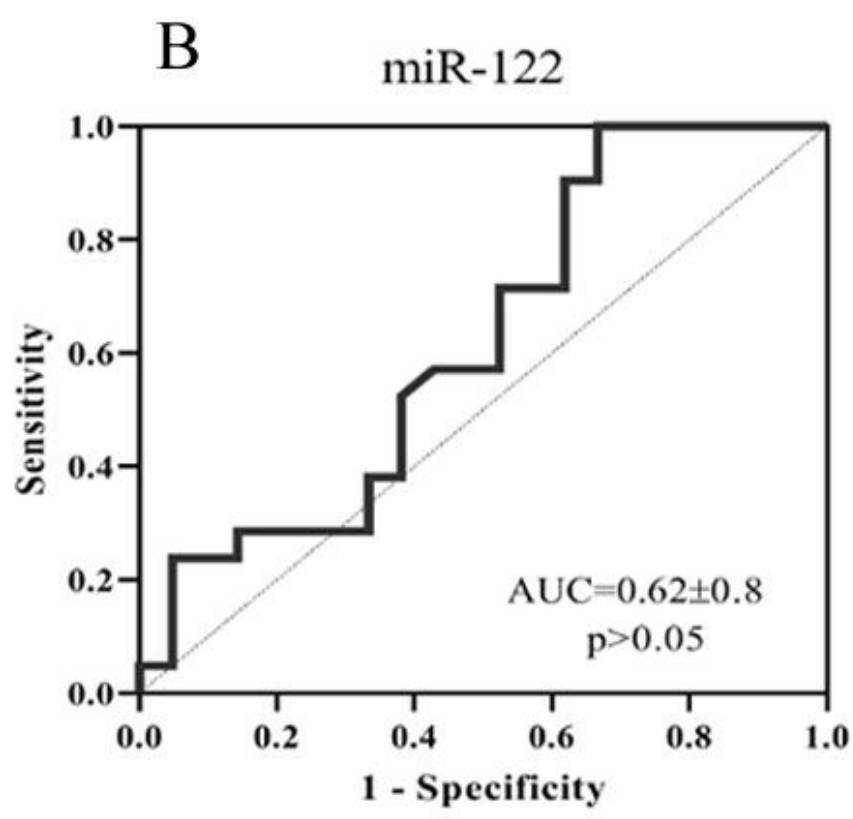

$\mathrm{D}$

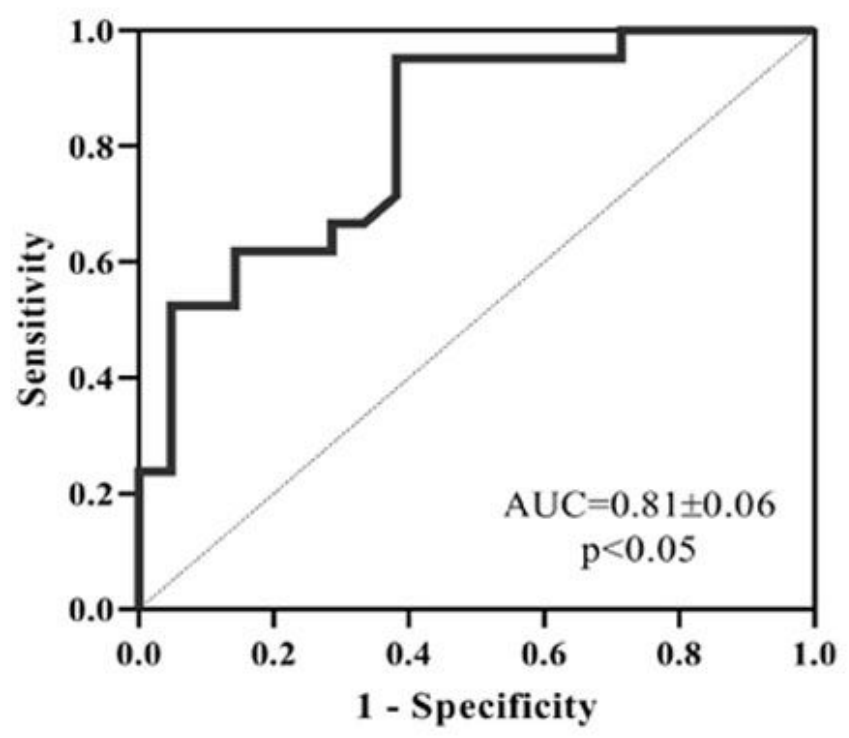

Figure 3

To distinguish the newly diagnosed ALL group from the control, an AUC for miR-22, miR-122, miR-217 and miR-367 was found to be 0.7 with a sensitivity of $61 \%$ and a specificity of $71 \%(P=0.03,95 \% \mathrm{Cl}: 0.69-0.94), 0.62$ with a sensitivity of $33 \%$ and a specificity of $100 \%(P=0.17,95 \% \mathrm{Cl}: 0.45-0.79), 0.81$ with a sensitivity of $95 \%$ and a specificity of $61 \%(P=0,95 \% \mathrm{Cl}: 0.69-0.94)$ and 0.8 with a sensitivity of $80 \%$ and a specificity of $81 \%(P=0.001,95 \%$ Cl: 0.65-0.94); respectively (Fig. 3A-D). 
A

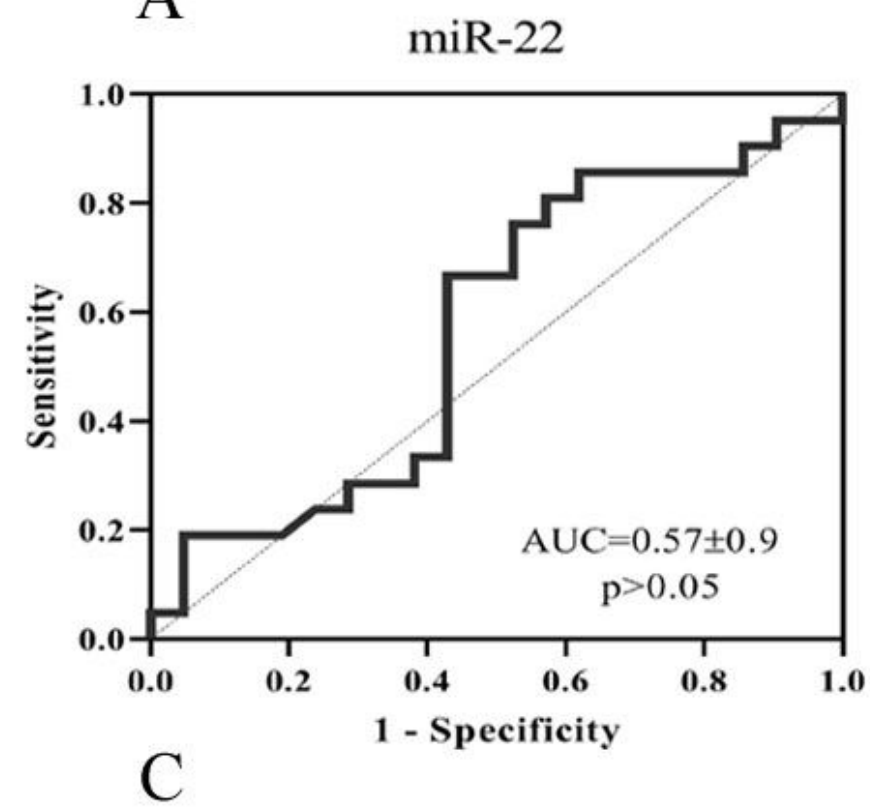

miR-367

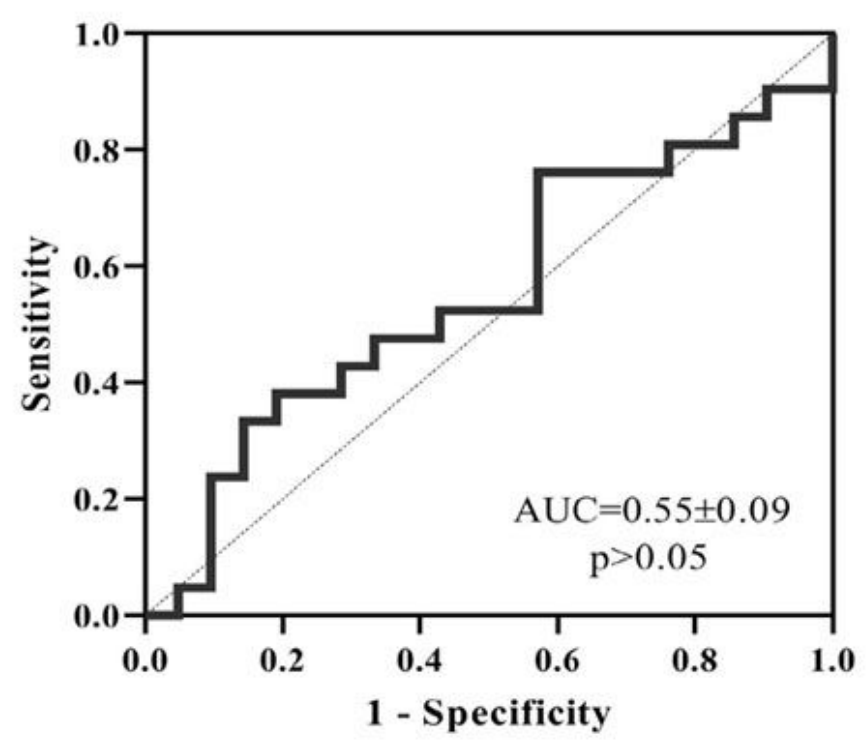

B

miR-122

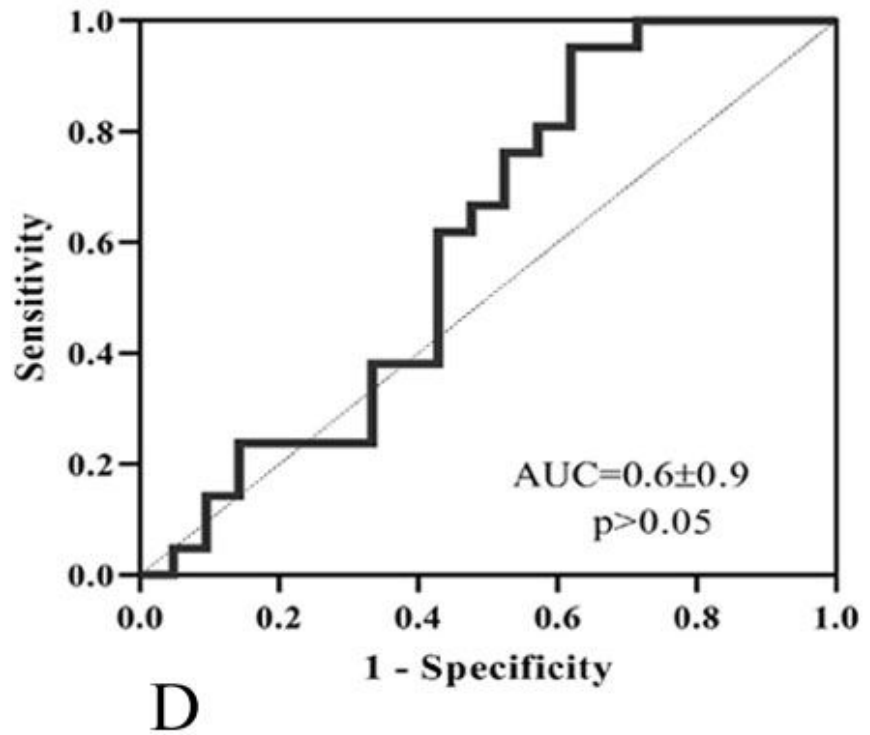

miR-217

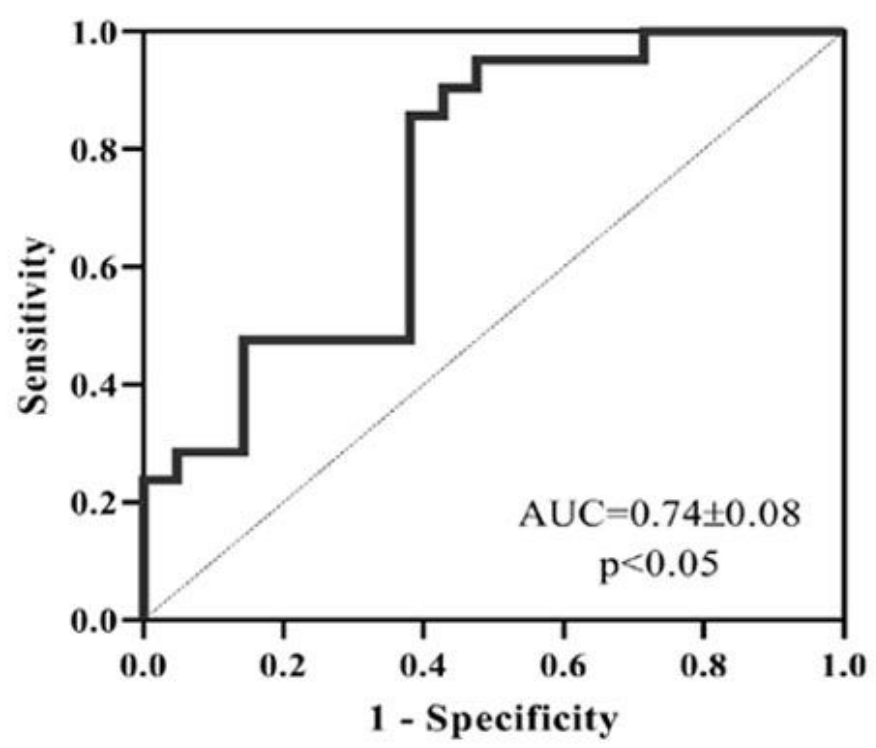

Figure 4

AUC in follow-up group for miR-22, miR-122, miR-217 and miR-367 was found to be 0.57 with a sensitivity of $38 \%$ and a specificity of $85 \%(P=0.43,95 \% \mathrm{Cl}: 0.39-0.74), 0.6$ with a sensitivity of $38 \%$ and a specificity of $95 \%(P=0.25$, $95 \% \mathrm{Cl}: 0.65-0.94), 0.65$ with a sensitivity of $80 \%$ and a specificity of $61 \%(\mathrm{P}=0.08,95 \% \mathrm{Cl}: 0.48-0.83)$ and 0.55 with a sensitivity of $42 \%$ and a specificity of $76 \%(P=0.53,95 \% \mathrm{Cl}: 0.37-0.73)$; respectively (Fig. $4 A-D)$. 
A

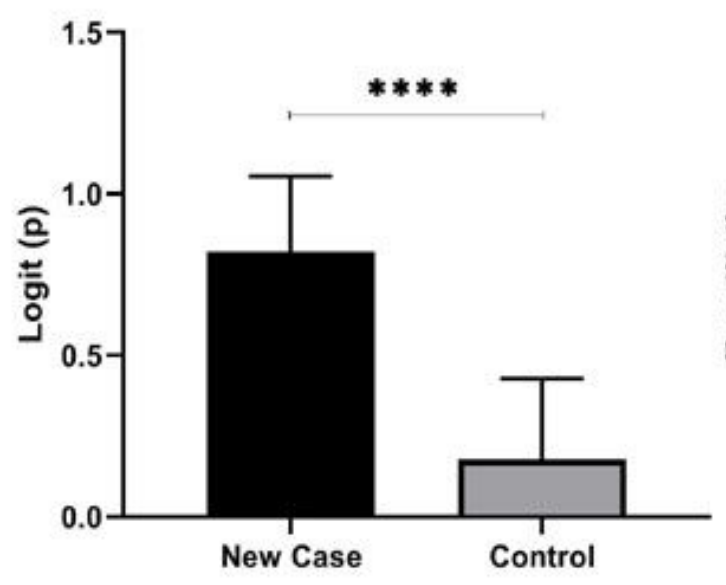

B

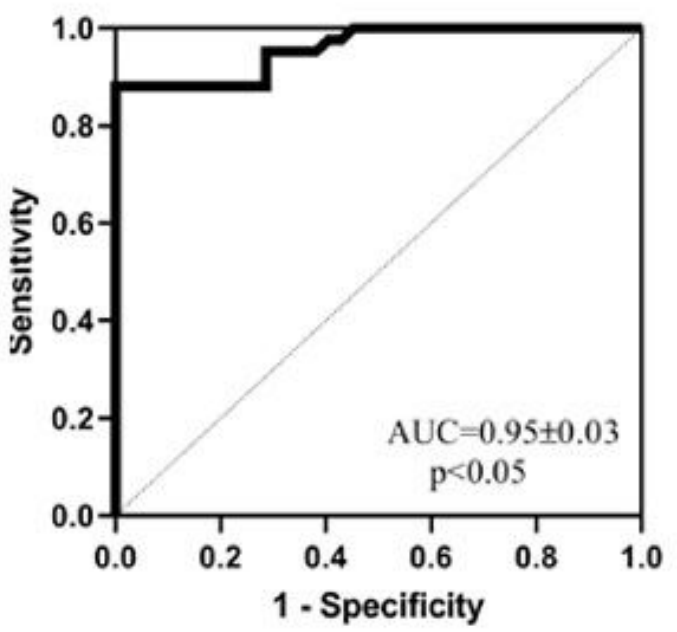

\section{Figure 5}

The predicted values of logistic regression analysis showed that the combined ROC analysis of these two miRNA revealed an increased AUC value of 0.95 , with a sensitivity of $95 \%$, a specificity of $90 \%$, and a Yuden index of 0.85 $(\mathrm{P}=0,95 \% \mathrm{Cl}:$ 0.88-1.01; Fig. 5). 